\title{
Therapeutic Approaches for Renal Colic in the Emergency Department: A Review Article
}

\author{
Samad EJ Golzari ${ }^{1}$; Hassan Soleimanpour ${ }^{2,}$; Farzad Rahmani ${ }^{3}$; Nahid Zamani Mehr ${ }^{4}$; Saeid \\ Safari $^{5}$; Yaghoub Heshmat ${ }^{2}$; Hanieh Ebrahimi Bakhtavar ${ }^{3}$ \\ ${ }_{1}^{1}$ Medical Philosophy and History Research Center, Tabriz University of Medical Sciences, Tabriz, Iran \\ ${ }_{3}^{2}$ Cardiovascular Research Center, Tabriz University of Medical Sciences, Tabriz, Iran \\ 3 Emergency Medicine Department, Tabriz University of Medical Sciences, Tabriz, Iran \\ 4 students \\ 5 Students Research Committee, Tabriz University of Medical Sciences, Tabriz, Iran \\ ${ }^{*}$ Corresponding author: Hassan Soleimanpour, Cardiovascular Research Center, Tabriz University of Medical Sciences, Tabriz, Iran. Tel: +989141164134, Fax: +984113352078, E-mail: \\ soleimanpourh@tbzmed.ac.ir
}

Received: November 17, 2013; Revised: December 8, 2013; Accepted: December 16, 2013

\begin{abstract}
Context: Renal colic is frequently described as the worst pain ever experienced, and management of this intense pain is necessary. The object of our review was to discuss different approaches of pain control for patients with acute renal colic in the emergency department. Evidence Acquisition: Studies that discussed the treatment of renal colic pain were included in this review. We collected articles from reputable internet databases.

Results: Our study showed that some new treatment approaches, such as the use of lidocaine or nerve blocks, can be used to control the severe and persistent pain of renal colic.

Conclusions: Some new approaches are discussed and their impact on renal colic pain control was compared with traditional therapies. The effectiveness of the new approaches in this review is similar or even better than in traditional treatments.
\end{abstract}

Keywords:Renal Colic; Lidocaine; Nerve Block; Emergency Department

\section{Context}

Urolithiasis has been known as a very common disease for centuries (1). Renal colic presents as acute renal colic pain in the flanks due to the passage of a stone from the ureter. The classic presentation of acute renal colic is a pain radiating from the flanks to the groin and accompanied by; microscopic hematuria ( $85 \%$ of patients), nausea, and vomiting. Another important finding is costovertebral angle tenderness $(2,3)$. In some cases, urinary infection, hydronephrosis, and continuous colic attacks have been observed in urolithiasis patients (4).

Approximately $8-15 \%$ of Europeans and North Americans will experience urolithiasis (5). A total of $12 \%$ of the population suffers from urolithiasis and about two million outpatient visits in the United States are related to kidney stones. In 2000, the economic cost of renal colic and urolithiasis was about $\$ 2.1$ billion (6). In $50 \%$ of people with a history of kidney stones, recurrence rates approach nearly $50 \%$ after 10 years. Kidney stone disease in men is 2-3 times more common than in women; in addition, it is more common in adults than in the elderly and it is least common in children. The disease is more common in white people, and in warm, dry climates. Decreased liquid intake and concentrated urine are two initial factors for the development of stones. Some drugs, such as; triamterene, indinavir, and acetazolamide, have been shown to be related to kidneys stones. Available oxalate in the food regimen is also categorized as another possible cause of kidney stones; although the role of the available calcium in dietary is less known and limiting the consumption of calcium in food regimens is not recommended in the long term (1). The risk of experiencing kidney stones in members of a urolithiasis patient's family is three times higher than in other individuals (7).

Renal colic pain emerges due to; obstruction of the urinary flow by a kidney stone, increased pressure on the urinary tract wall, smooth muscle spasms of the ureter, edema and inflammation near the stone, increase in peristalsis, and pressure of the proximal stone $(8,9)$. Increased pressure

Implication for health policy/practice/research/medical education:

In the present review, new treatment approaches for renal colic are discussed, such as the use of lidocaine or nerve blocks, which can be used to control severe and persistent renal colic pain in the emergency department.

Copyright (C) 2014, Iranian Society of Regional Anesthesia and Pain Medicine(ISRAPM); Published by KowsarCorp. This is an open-access article distributed under the terms of the Creative Commons Attribution License, which permits unrestricted use, distribution, and reproduction in any medium, provided the original work is properly cited. 
in the urinary tract following an increase of pressure in the local blood flow, and urethral smooth muscle contractions, are the main mechanisms of pain in these patients; furthermore, there is increased sensitivity to pain (10). Tension in the renal pelvis leads to the stimulation, synthesis and local release of prostaglandins, which induces diuresis and vasodilation, which in turn increases intrarenal pressure. The direct effect of prostaglandins on the ureter causes spasms in the smooth muscles of the ureteral wall (8). Permanent obstruction of the urinary tract due to renal stones leads to the release of prostaglandins in response to the existing inflammation. Within the initial hours following an obstruction, the gradient pressure between the renal glomeruli and the renal pelvis becomes equal, and consequently the glomerular filtration rate and renal blood flow decrease. If ureteric obstruction is not resolved, renal failure can occur. The best and most effective treatments for the pain of renal colic are; the spontaneous passage of a urethral stone, stone removal, placement of a stent in the ureter, and percutaneous nephrostomy. Fortunately, most patients do not suffer from complete ureter obstruction and thus they do not face the risk of renal failure (9).

Renal colic pain is often described as the worst pain the patient has ever experienced (2,11-13). Consequently, the use of effective pain killers like; non steroidal anti-inflammatory drugs (NSAIDS), and opioids, or a combination of medications (anti-inflammatory and spasmolytic agents), play important roles in the treatment of these patients. Recently, the use of alpha-blockers (due to decreases in the transit time of urinary tract stones), nifedipine, intravenous lidocaine and nerve blocks in the paravertebral region, are known to reduce pain in renal colic $(3,5,11)$.

The majority of family physicians have a lot of experience in the treatment of renal colic during their clinical practice. In recent years, great progress has been made in detection technologies of kidney stones. Nowadays, doctors can detect a renal stone to approve or reject within minutes after confirming the diagnosis. Furthermore, when the diagnosis of urolithiasis has been made, there are clear indications for a patient's referral to a urologist(1).

\section{Evidence Acquisition}

Articles used in this review were accessed from available evidence on approaches to renal colic treatment in emergency departments. The following key words were used: Therapeutic approaches; Treatment; Renal colic; and Emergency department. We searched for systematic reviews, evidence-based clinical practice guidelines, health technology assessments, and randomized controlled trials. In addition, in order to achieve a better conclusion we used the following data bases and sites:

1) Cochrane Library

2) Medline (Ovid)

3) PubMed

In this paper we only reviewed articles published from
1998 up to 2013, and our criteria for inclusion and exclusion were as follows:

Inclusion Criteria:

-Studies on renal colic treatment

-Studies performed in the adult age group

Exclusion Criteria:

-Studies published in a language other than English

\subsection{Analysis}

The search strategy resulted in 482 articles. A title review excluded irrelevant papers (348) leaving 138 articles. Fifty seven articles were selected for further analysis including: 25 Randomized Clinical Trial, 13 review articles, 6 observational studies, 4 systematic reviews, 3 cohort studies, 2 books, 2 short reports, 1 case report, and 1 case series, which met our criteria (Figure 1). The remaining 81 articles were excluded due to the following reasons: irrelevant abstracts or full-text reviews (18 articles), duplicate records (15 articles), reviews (15 articles), and studies on renal colic without treatment (48 articles). The characteristics of all the included studies are shown in Table 1. In addition, categorizations and the number of reviewed papers are shown in Table 2.

\section{Results}

Therapeutic approaches for the treatment of renal colic in the emergency department were introduced in the studies. However, in the studies; a common point of treatment for renal colic in the emergency department was the rapid and complete control of renal colic pain. In the following section, we investigate and explain the different types of renal colic approaches in emergency departments (Table 1 ).

\subsection{Opiates}

Narcotics have long been used for pain control in renal colic (5-7). Although narcotics such as morphine, codeine and meperidine for pain relief in patients with renal colic are effective, they have little effect on the underlying cause of renal colic (prostaglandins)(1). The benefits of using opioids include; low cost, good effect and titration possibility. However, the majority of physicians are not comfortable with using these drugs due to their side effects which include; nausea, vomiting, sedation, dizziness, lightheadedness, narcotic dependence, disorientation, respiratory depression, and hypotension $(6,7)$. Opiates can be administered in different ways, but the intravenous form is preferred because of its rapid onset. Among the different types of narcotics, morphine is used most frequently because it is stronger and less addictive than meperidine. On the other hand, there is not enough data on the effect of opiates on ureter muscle tone and there are certain conflicts; some data suggest that ureter smooth muscle tone increases with opiates, yet others suggest no effect of opiates on ureter smooth muscle tone (7-9). 
Golzari SE et al.

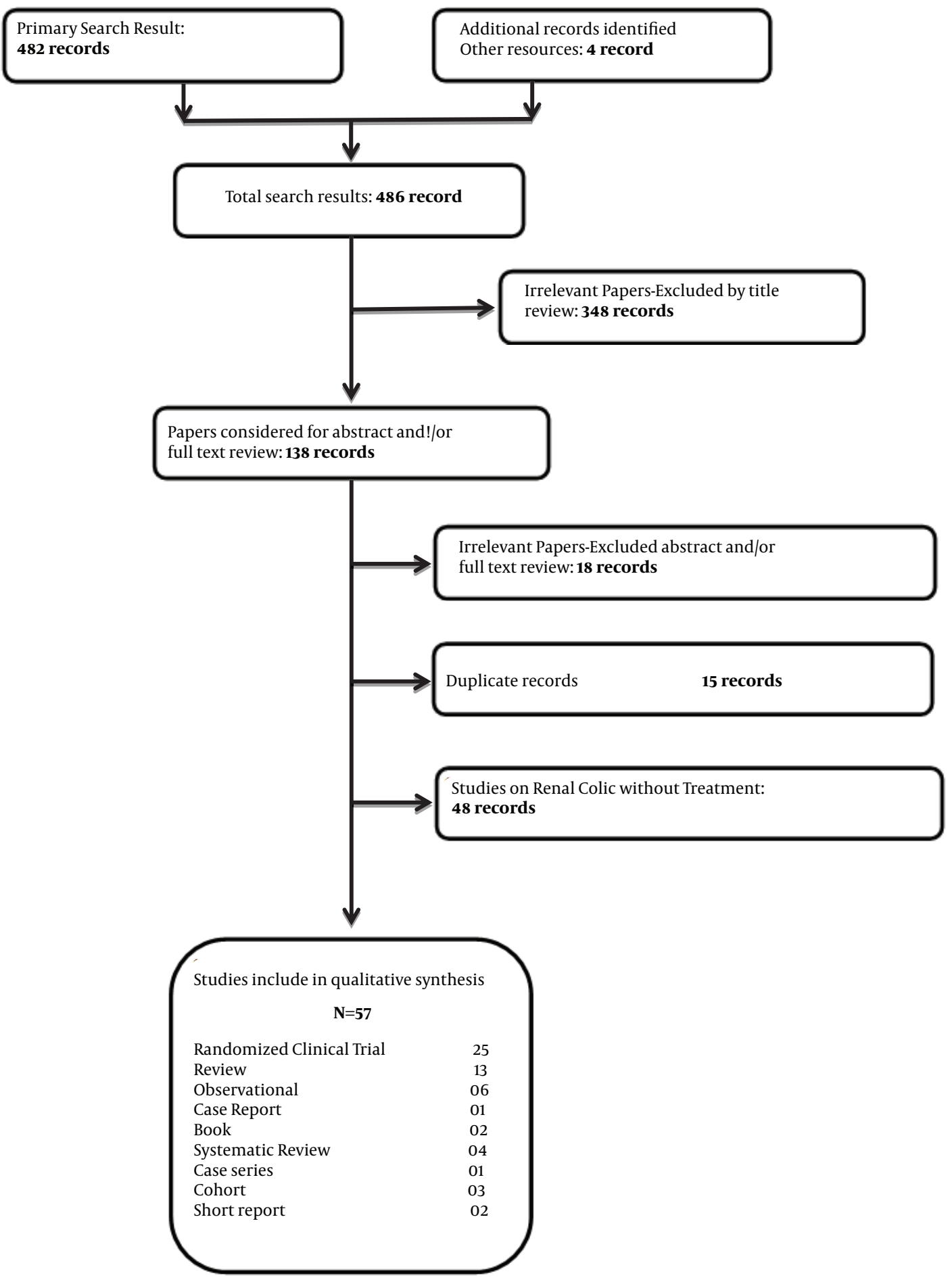

Figure 1. Flow Diagram of Research Papers in Our Study 
Golzari SE et al.

\begin{tabular}{|c|c|c|c|}
\hline Drug & Administration Route & Study & Year \\
\hline \multirow[t]{2}{*}{ Lidocaine } & Intravenous & Soleimanpour et al. $(3,32)$ & $2012 ; 2011$ \\
\hline & & Ferrini et al. (31) & 2004 \\
\hline \multirow[t]{24}{*}{ NSAIDs } & Oral & Holdgate et al. $(4,8)$ & $2005 ; 2004$ \\
\hline & & Phillips et al.(6) & 2009 \\
\hline & & Davenport et al. (15) & 2010 \\
\hline & & Supervia et al.(16) & 1998 \\
\hline & & Cohen et al. (19) & 1998 \\
\hline & & Lafrance et al. (21) & 2009 \\
\hline & & Bleumink et al. (22) & 2003 \\
\hline & & Kearney et al. (23) & 2006 \\
\hline & & Grissa et al.(28) & 2011 \\
\hline & & Sakhare et al. (38) & 2012 \\
\hline & & Sumer et al. (51) & 2012 \\
\hline & Intravenous & Safdar et al. (7) & 2006 \\
\hline & & Bektas et al. (12) & 2009 \\
\hline & & Davenport et al. $(9,15)$ & $2005 ; 2010$ \\
\hline & & Cohen et al. (19) & 1998 \\
\hline & & Larkin et al. (20) & 1999 \\
\hline & & Serinken et al. $(10,24)$ & $2008 ; 2012$ \\
\hline & & Morgan et al.(25) & 2011 \\
\hline & & Duggan et al.(26) & 2009 \\
\hline & & Gorocs et al. (27) & 2009 \\
\hline & & Grissa et al.(28) & 2011 \\
\hline & & Lee et al. (29) & 2010 \\
\hline & & Song et al. (36) & 2012 \\
\hline & & Kekec et al. (48) & 2000 \\
\hline \multirow[t]{12}{*}{ Opioids } & Intravenous & Soleimanpour et al. (3) & 2012 \\
\hline & & Holdgate et al. $(2,4,8)$ & $2004 ; 2005$ \\
\hline & & Safdar et al. (7) & 2006 \\
\hline & & Bektas et al. (12) & 2009 \\
\hline & & Hazhir et al. (14) & 2010 \\
\hline & & Lee et al. (29) & 2010 \\
\hline & & Asgari et al. (42) & 2012 \\
\hline & & Snir et al. (43) & 2008 \\
\hline & & Yencilek et al. (44) & 2008 \\
\hline & Intramuscular & Hazhir et al.(14) & 2010 \\
\hline & & Larkin et al. (20) & 1999 \\
\hline & & Song et al. (36) & 2012 \\
\hline \multirow[t]{2}{*}{ Phloroglucinol } & Oral & Dellabella et al. (5) & 2005 \\
\hline & & Boubaker et al. (37) & 2010 \\
\hline \multirow[t]{4}{*}{ Conventional and Alternative Methods } & NA & Davenport et al.(9) & 2005 \\
\hline & & Nuss et al. (52) & 2005 \\
\hline & & Kober et al.(56) & 2003 \\
\hline & & Soleimanpour et al. $(3,32)$ & 2012; 2011 \\
\hline
\end{tabular}


Golzari SE et al.

\begin{tabular}{llll}
\hline Trigger Point Injection & NA & Iguchi et al. (11) & 2002 \\
\hline Hyoscine Butylbromide & Intramuscular & Samuels et al. (33) & 2009 \\
\hline Drotaverine Hydrochloride & & Kheirollahi et al. (34) & 2010 \\
& & Song et al. (36) & 2012 \\
\hline Aminophylline & & Sakhare et al. (38) & 2012 \\
\hline & Intravenous & Palea et al. (39) & 2006 \\
\hline Glycerol Trinitrate & & Romics et al. (40) & 2003 \\
\hline Nifedipine & & Barzegarnezhad et al. (45) & 2012 \\
\hline Alpha1-Adrenergic Blockers & Nickels et al. (46) & 2006 \\
\hline & Topical & Djaladat et al. (47) & 2007 \\
\hline & Oral & Hussain et al. (49) & 2001 \\
\hline
\end{tabular}

Table 2. Categorizations and Number of Reviewed Papers

\begin{tabular}{llllllll}
\hline Books & $\begin{array}{l}\text { Original } \\
\text { Articles }\end{array}$ & $\begin{array}{l}\text { Article Re- } \\
\text { views }\end{array}$ & $\begin{array}{l}\text { Systematic } \\
\text { Reviews }\end{array}$ & CaseControls & Case Series & Case Reports & $\begin{array}{l}\text { Short Re- } \\
\text { ports }\end{array}$ \\
\hline $\mathbf{2}$ & 31 & 13 & 4 & 3 & 1 & 1 & 2 \\
\hline
\end{tabular}

Codeine and dihydrocodeine are weaker than morphine, but will relieve mild to moderate pain. Constipation is one of the main side effects of these drugs, so the long-term use of these drugs is limited. The potency of dextropropoxyphene is half that of codeine, but it can be used in combination with paracetamol (co-proxamol) for the treatment of mild pain (in contraindications of opioids). Little evidence is available on the superiority of this combination over paracetamol $(9,12)$.

Tramadol is another narcotic with has fewer potential side effects such as; respiratory depression, constipation, or dependence, compared to other opiates. Tramadol is as effective as morphine in reducing moderate pain after surgery, but it is less effective in more severe pain. Common side effects of this drug include; lightheadedness, nausea, dry mouth and sedation (9). Hazhir et al. evaluating the effect of intramuscular tramadol and meperidine, concluded that the effect of $100 \mathrm{mg}$ tramadol is similar to the effect of $50 \mathrm{mg}$ of pethidine. Nevertheless, further studies are required to confirm the therapeutic effect of tramadol in reducing pain in renal colic patients compared to previous treatments (14).

\subsection{Non-Steroidal Anti-Inflammatory Drugs (NSAIDs)}

NSAIDs alone or in combination with opioids have been used to treat renal colic pain for a long time (6). Analgesic effects of these medications are due to the inhibition of prostaglandin synthesis. As a result, NSAIDs prevent afferent arterial vasodilation and increase vascular per- meability, which cause diuresis and increased pressure within the renal pelvis. NSAIDs also reduce edema, inflammation and ureter muscular hyperactivity (10).

The effect of NSAIDs on relieving pain in acute renal colic is similar to opiates. The only disadvantage of NSAIDs, in the oral or rectal form, is the delayed onset time compared with intravenous morphine. Intravenous forms of NSAIDs are available and have a rapid onset, but side effects from the intravenous form of NSAIDs have been reported more frequently than for other types of drugs. Complications of NSAIDs include; nausea, vomiting, feeling of heat or pressure in the chest, fatigue and lethargy (15).

In a meta-analysis by Holdigate et al. it was indicated that the patients for whom NDAIDs were prescribed; require less medication for pain control, experience less nausea, and have greater improvements in their pain (8).A fast-dissolving dosage form of piroxicam is similar to intramuscular sodium diclofenac in reducing pain in patients with renal colic. Moreover, due to the ease of sublingual piroxicam use, medication compliance by patients is increased (16).

In a randomized clinical trial by Phillips et al. the effect of celecoxib in the treatment of acute renal colic was studied. Patients were treated with celecoxib $400 \mathrm{mg}$, then $200 \mathrm{mg}$ every 12 hours for 10 days, and then compared with a placebo-treated group. Later, it was concluded that celecoxib had no effect on the stone canal passage, but it reduced analgesic requirements (6).

During extracorporeal shock wave lithotripsy (ESWL), 
NSAIDs are frequently used for pain control. In their study, Labanaris et al. found that aspirin increases the risk of bleeding and prerenal hematoma (especially if accompanied by uncontrolled hypertension) during this treatment; however, no evidence of increased bleeding risk was reported with other NSAIDs (17).

Gastrointestinal side effects of NSAIDs are due to the inhibition of gastric mucosal protective prostaglandin synthesis and mucosal damage caused by stomach acid. The use of long-acting and slow release forms of NSAIDs increases the risk of upper gastrointestinal bleeding. The relative risk of upper gastrointestinal bleeding caused by NSAIDs is about 4.5 (3.8-5.3). The risk in ibuprofen (2.6),when compared with ketorolac (14.54), is obviously low,while indomethacin is 5.4 and diclofenac is 3.98 (18).

Cohen et al. in their review,' A Comparison in Effect of Diclofenac and Ketorolac in the Treatment of Renal Colic,' came to the conclusion that there is no significant difference in efficacy between the two drugs in pain relief of patients with renal colic (19). On the other hand, in a comparison with intramuscular ketorolac (60 mg) and intramuscular meperidine (150-100 mg) by Larkin et al. it was concluded that ketorolac has a better effect in reducing patients' renal colic pain than meperidine (20). In addition, when administered together, intravenous morphine and ketorolac reduce pain better than either drug does alone (7).

NSAIDs also interfere with the auto-regulatory renal blood flow system and reduce renal blood flow. This effect of NSAIDs is well-tolerated in healthy subjects without renal disease, but in patients with; previous renal disease, dehydration, cirrhosis, and recent use of nephrotoxic drugs or contrast agents, they can induce renal failure. Prostaglandins cause vasodilation in afferent glomerular arteries, and play a vital role in normal glomerular perfusion and glomerular filtration rates (GFR). As NSAIDs inhibit the synthesis of prostaglandins, they lead to a contraction of afferent arteries and a reduction in renal perfusion pressure. Nausea and vomiting are often seen in patients with renal colic which can lead to dehydration and may further contribute to renal impairment (15). Lafrance et al. in their study on the risk of renal failure due to selective and non-selective nonsteroidal anti-inflammatory drugs, came to the conclusion that the risk of acute renal failure in patients taking selective cyclophosphamide oxygenase (COX) enzyme inhibitors is lower than in non-selective agents and (COXII) enzyme inhibitors; therefore, diclofenac, ibuprofen, naproxen and ketorolac are less risky for renal failure (21).

In patients with previous heart disease, the use of NSAIDs may lead to the development of heart failure and cardiac decompensation, due to increased peripheral vascular resistance and decreased renal perfusion, in patients with impaired ventricular function and compensatory increased vasodilator prostaglandins (15). In a review of NSAIDs and heart failure, Bleumink et al. con- cluded that a reduction in renal blood flow, glomerular filtration, and sodium excretion, increased the load on fluids and increased systemic vascular resistance, which may contribute to the risk of kidney failure in susceptible patients (22).

The risk of coronary events increases in NSAIDs consumers. COX II inhibitors increase the cause of coronary events and myocardial infarction by $25 \%$. High-dose ibuprofen (800 mg 3 times daily) and high-dose diclofenac (75 mg 2 times daily) have also been associated with the risk of coronary events; however, naproxen (500 mg 2 times a day) risk in causing a coronary event is lower. It is assumed that this effect is due to an inhibition of platelet aggregation (23).

\subsection{Alternative Treatments}

\subsubsection{Paracetamol}

Paracetamol (acetaminophen) is a safe and effective analgesic with fewer side effects than NSAIDs and opiates. The drug can be administered orally, rectally or intravenously (12). Despite 50 years of research conducted on acetaminophen, the actionmechanism of this drug has still not been fully characterized (24). However, due to significant concentrations in the cerebrospinal fluid after administration of the drug, it is believed that this drug works on the central nervous system (25). Acetaminophen acts by inhibiting prostaglandin synthesis (which are free of inflammatory response) (26), furthermore, metabolites of acetaminophen with $\mathrm{N}$-arachidonoylaminophenol (AM404) inhibit endogenous cannabinoids, such as an andamide reuptake in the synaptic cleft, and consequently they cause analgesic effects (24). Intravenous, compared with oral or rectal acetaminophen, has a more rapid onset time thanks to its direct entry into the bloodstream (27). Acetaminophen is well-tolerated and side effects are rare, but it can cause; weakness, hypotension and elevated liver enzymes (26). In patients with renal failure because of delayed drug elimination, the drug half-life increases to five hours; the recommended interval between the administration of the drug in these patients is six hours (25). Bektas et al. in their study comparing the effects of paracetamol and morphine on renal colic pain relief concluded that the effect of intravenous acetaminophen in pain reduction is much better and it has fewer side effects than intravenous morphine (12). Grrisa et al. in another study comparing the effects on renal colic in pain relief between paracetamol and piroxicam, suggested that pain relief following the administration of a single dose of intravenous paracetamol was superior to intramuscular piroxicam (28). Lee et al. in their study on the comparative effects of paracetamol and morphine for the treatment of pain after thyroidectomy, concluded that the effect of $1 \mathrm{~g}$ of intravenous paracetamol is similar to $30 \mathrm{mg}$ of ketorolac on pain 
relief, so when NSAIDs are contraindicated, intravenous paracetamol could be an option (29).

\subsubsection{Lidocaine}

Lidocaine has become the agent of choice in visceral and central pain. On the other hand, when narcotics are not effective, the administration of lidocaine is a useful alternative and there are no unwanted side effects similar to that of other narcotics. Intravenous lidocaine is effective in the management of neuropathic pain such as; diabetic neuropathy, post-surgical pain, post herpetic pain, headaches, and neurological malignancies (3). Lidocaine is an anesthetic amide that reversibly blocks voltage-dependent sodium channels and thus it may lead to the inhibition of nerve impulse transmissions. Systemic toxicity associated with the administration of lidocaine occurs because it blocks sodium channels in the heart and brain. Poisoning symptoms can range from; mild neurologic symptoms, to intractable seizures, and cardiovascular collapse (30). However, if administered at low doses, lidocaine is a relatively safe medication. Allergy to lidocaine leads to an increased risk of cardiac dysrhythmias and dyspnea in some patients. Lidocaine is effective, inexpensive and has few side effects including; lightheadedness, nausea and constipation. Overall, the incidence of side effects compared to those of other drugs and narcotic analgesics is low. On the other hand, a wide range of adverse effects of lidocaine are predictable, but due to the lower half-life of lidocaine, lidocaine toxicity symptoms are transient and rapidly reversible (3).

Ferrini et al. in their review on the use of lidocaine in the treatment of severe pain or neuropathic pain, concluded that lidocaine is more effective in reducing visceral and central pain compared to narcotics. Furthermore, when the use of narcotics is not effective or side effects are observed in patients, lidocaine is appropriate (31-32). Soleimanpour et al. in their study compared the effects of intravenous lidocaine and morphine in the treatment of renal colic in the emergency department and found that lidocaine significantly reduces pain compared to morphine in patients with renal colic (3).

\subsubsection{Hyoscine Butyl Bromide}

Anti-muscarinic agents are effective in the treatment of smooth muscle spasms (especially gastrointestinal). Ureteral peristaltic activity of the genitourinary system is controlled by the autonomic nervous system so the use of anti-muscarinic agents can be effective (9). Hyoscine butyl bromide (Buscopan TM) is an anti-muscarinic drug which blocks the action of acetylcholine at the parasympathetic nerve endings in muscles and glands (33), and theoretically it is effective when administered in relieving pain associated with analgesic drugs for moderate renal colic pain (2).
Holdgate et al. in their review on the role of anti-muscarinic agents (hyoscine butyl bromide) in renal colic, reported that Buscopan has no effect in reducing renal colic pain and it does not reduce the need for additional opiates (2). In their study, Kheirollahi et al. compared the effect of combined intranasal desmopressin and intramuscular hyoscine with hyoscyamine alone in acute renal colic, and they found that the combination of hyoscine with desmopress in alone is more effective in renal colic (34). Kheirollahi et al., in their study comparing the effect of hyoscine with diclofenac and diclofenac alone in the treatment of renal colic, concluded that the combination of diclofenac and hyoscine compared with diclofenac alone, resulted in rapid improvement of renal colic pain (34). Song et al. in their review on the effect of adding hyoscine to ketorolac and morphine in patients with renal colic, stated that the combination of morphine and hyoscine with ketorolac creates a greater reduction in patients' pain. This decrease is statistically significant, but clinically the reduction in pain intensity is not significant (36).

Hyoscine butyl bromide has obvious side effects including: dry mucous membranes, photophobia, facial flushing, dry skin, loss of accommodation, constipation, urinary retention, and renal colic thus limiting its routine administration (9,35-36).

\subsubsection{Phloroglucinol}

Phloroglucinol is a drug with potent antimuscarinic effects that are well-tolerated (37), although little evidence about the effectiveness of these drugs is available for renal colic. In their study, Boubaker et al. evaluated the analgesic effect of this drug in combination with piroxicam on renal colic, it was suggested that phloroglucinol has no effect in reducing the severity of pain when added to piroxicam (37).

\subsubsection{Drotaverine}

Drotaverine is an inhibitor of Phosphodiesterase 4 (PDE4) in smooth muscles and it has anti-spasmodic activity without anti-muscarinic adverse effects. This drug is structurally similar to papaverine and its structural formula is Diethoxy-1, 2, 3, 4-tetrahydroisoquinoline 1 - (3, 4-diethoxybenzylidene) -6, 7 (38). Drotaverine provides effective analgesia and is used in the treatment of renal colic (39). Palea et al. in their review on the effects of drotaverine relaxation on the human urethral muscle ring, found that the effect of drotaverine relaxation on the muscles of the humanureter is similar to that of other Animal and its power is approximately six times stronger than papaverine: hence, the ureteral muscle relaxant advantage can be used in the treatment of renal colic patients (39). Romics et al. in their study on the impact of drotaverine on renal colic pain, reported that pain intensity was reduced in more than two-thirds of patients 
with intravenous drotaverine. Drotaverine's side effects are not significant, but include; transient drop in blood pressure, dizziness, nausea and vomiting (40).

\subsubsection{Papaverine Hydrochloride}

Papaverine,1-[(3,4-dimethoxyphenyl) methyl]-6,-7-dimethoxyisoquinoline, results in smooth muscle relaxation and is found in opiate compounds. Due to the relaxation properties of this drug, it is used in an intra-arterial injection, as a vasodilator in cerebral vasospasm after a subarachnoid hemorrhage. Furthermore, it is also used as a therapeutic agent in erectile dysfunction (impotence) and renal colic. Its pharmacological effects are due to blocking of the voltage-dependent potassium channels (41).

Asgari et al. in their review on the effects of papaverine in the treatment of renal colic, concluded that adding papaverine to diclofenac, compared with diclofenac alone, has a greater impact in reducing pain. The only reported side effect of papaverine is dizziness, which occurs in only a small percentage of patients (2\%) (42). Snir et al. concluded that using papaverine in the treatment of renal colic is as effective as sodium diclofenac in the management of patients with renal colic pain in the short term, and can be effective if there are contraindications for the use of NSAIDs; however, the analgesic effect of diclofenac in controlling pain lasts longer than papaverine (43). Yencilek et al. reported that papaverine is more effective in renal colic pain relief in patients with refractory pain than conventional agents (44).

\subsubsection{Aminophylline}

Aminophylline is a methylxanthine drug and a derivative of theophylline. The strength and duration of aminophylline's action is less than theophylline. It relaxes smooth muscles, especially; muscles of the bronchial walls, heart and central nervous system stimulation, and diuresis. This drug also crosses the placental barrier. Its mechanism is through the inhibition of a non-selective phosphodiesterase inhibitor that; increases the concentration of intracellular cAMP, activates PKA (protein kinase A), inhibits TNF- $\alpha$ and the synthesis of leukotrienes, and reduces inflammation. Non-selective antagonism of adenosine receptors is the second mechanism (45). Aminophylline is a wellknown drug and it is widely used in the treatment of renal colic (46).

Djaladat et al. in their study on the effect of aminophylline on renal colic, concluded that aminophylline decreases renal colic pain in patients and reduces the need for a narcotic prescription (47). In their study using a local injection of aminophylline during trans-urethral lithotripsy, Baregarnezhad et al. showed that aminophylline facilitates the uretroscopy procedure, increases the success rate for the treatment of renal colic by using (transurethral lithotripsy), decreases operative time, and reduces the need for a double J catheter and wave lithotripsy (45).

\subsubsection{Nitrates}

Nitrates, thanks to their impact on vascular smooth muscle, have been studied in the treatment of renal colic. Two surveys have been conducted on the effects of isosorbide dinitrate and glycerol trinitrate (9). Nitrates in vascular smooth muscle lead to the release of nitric oxide and ultimately to increased concentrations of cGMP, which interferes with guanylyl cyclase and the relaxation of smooth muscles. This effect is caused by nitrates in the genital tract smooth muscle. However, due to the short effectiveness time of nitrates, the clinical value of smooth muscle relaxant effects in the genitourinary system is low (48). Little evidence is available on the effect of these drugs on renal colic.

Kekec et al. studied the effect of adding NSAIDs to isosorbide dinitrate in the treatment of renal colic and came to the conclusion that adding isosorbide to tenoxicam, compared with tenoxicam alone, results in a decrease in pain severity, and this reduction in pain intensity is clinically significant. Side effects observed in this study were; headaches, flushing and orthostatic hypotension (48).

Hussain et al. in their study on the effects of glycerol trinitrate patches (GTN) in patients with ureteral stones with a diameter less than $10 \mathrm{~mm}$ over a period of six weeks, concluded that patients who used the GTN patches experienced less pain episodes compared with placebo groups; however, the rate was not statistically significant. During the treatment period, $27 \%$ of the patients discontinued their treatment with GTN because of headaches (49).

\subsubsection{Calcium Channel Blockers}

In-vitro studies have shown that calcium channel blockers decrease ureteral human peristalsis. Calcium is essential for the maintenance of action potential in the ureter ductand also for contraction of the ureter. Calcium channel blockers inhibit the influx of calcium, and therefore are expected to have an inhibitory effect on the activity of the ureter. Nifedipine appears to be the most effective drug in this category $(5,9)$. Little evidence for the effectiveness of nifedipine in acute renal colic or its role in facilitating the passage of duct stones is available.

In a study by Porpiglia et al. (50), it was found that although nifedipine does not reduce pain in the acute phase, it can lead to stone removal from the channel in a short time and decrease analgesic requirements. Due to their anti-inflammatory effects, steroids are administered with nifedipine; although they are effective, the risks of long-term steroids use should be considered.

\subsubsection{Alpha-Blocker}

Ureteral activity is regulated by the autonomic nervous system. In the sympathetic nervous system, $\alpha$-fibers are 
stimulants and $\beta$-adrenergic inhibitory (9), in addition, $\alpha-1$ adrenergic receptors are available in the human ureter. These receptors, especially subgroup $1 \mathrm{D} \alpha$, play roles in the dilatation of detrusor muscles and spasms of the distal third of the ureter (51). Blocking alpha-adrenergic receptors reduces spasms and pain, and in addition it induces ureteral stone removal (52).

Yilmaz et al. treated renal colic in patients who had distal ureteral stones with alpha-adrenergic blockers, and they found that the use of these drugs facilitates the removal of ureteral stones (53). Parsons et al. in their study on patients with ureteral stones, showed that alpha-blockers may lead to increased rates of urethral stones removal (54). Sumer et al. divided patients with urolithiasis into three groups, and treated each group separately with; diclofenac and alpha-blocker combination, diclofenac and prednisolone combination, and the third group received diclofenac alone. In the group treated with adiclofenac and alphablocker combination, stone removal rates were higher in comparison with the other two groups. Alpha-blocker drugs are used more often in combination with antibiotics and oral corticosteroid therapy for medical expulsive therapy (MET) (51).

MET is considered in the treatment of patients with uncomplicated distal ureteral stones before uretroscopy or extracorporeal lithotripsy. This method increases the success rate of stone removal and decreases ureteral colic. In newly-diagnosed patients with ureteral stones less than $10 \mathrm{~mm}$ in diameter, the symptoms are controlled; however, periodic assessment and observation of patients is necessary. In these patients, the use of MET is considered in order to facilitate the removal of duct stones. There is evidence that MET reduces the need for the administration of analgesia and accelerates the passage of ureteral stones with diameters less than $10 \mathrm{~mm}$ (similar size pieces of stone after shock wave lithotripsy (SWL)). Evidences suggest that MET could be considered as an effective treatment (51).

Dellabella et al. have suggested that prescribing tamsolosin results in stone removal in almost all patients in a very short time, without the need for hospitalization. In another research on the effects of tamsolosin on drug treatment of juxtavesical ureteral stones, it was concluded that the use of tamsolosin in patients increases stone removal rates, decreases the need for hospitalization and endoscopic procedures, as well as providing better control of the patients' colic pain (55).

\subsubsection{Trigger Point Injection}

Trigger point injection (TPI) has been used to control pain in patients with chronic, visceral, and myofascicular pain. In patients with renal colic, a local anesthetic is injected into the ipsilateral posterior surface of the trigger points. In this method, the patient is placed in a prone position andgentle pressure is used over the trigger points with the end of a ballpoint pen, and then markedat intervals of $1 \mathrm{~cm}$ in the triangular area bounded by the edges of the ribs, spine and iliac crest. When pressure is applied and the patient feels pain, the trigger points are determined. Subsequently, 2-3 mL of lidocaine $1 \%$ is injected into an area of about $3 \mathrm{~cm}$ in diameter, and using a long (23 gauge, 6 $\mathrm{cm}$ ) needle, 5-10 $\mathrm{mL}$ of $1 \%$ of lidocaine is injected into the deep portion of the psoas muscle $(3,11)$. Iguchi et al. used this method for pain control in patients with renal colic, and concluded that the combination of sulpyrine and butyl scopolamine was more effective and resulted in faster pain control. This method is also simple, effective and safe (11). Other alternative techniques such as subcutaneous paravertebral nerve block, and sympathetic chain block and catheter insertion into the upper lumbar sympathetic chain for continuous infusion, have also been used successfully in the treatment of renal colic $(3,11)$.

\subsection{Non-Medicated Alternative Therapies}

\subsubsection{Local Active Warming}

Local heating has been suggested as a way to reduce pain in trauma patients in the emergency department. Localized heating of the active region of the abdomen and lower back can be a simple and effective method for pain control in patients with suspected renal colic during patient transportation to the emergency department and hospital. Kober et al. reported that local heating in the abdominal and back areas of patients with renal colic, significantly decreases anxiety and pain (56).

\subsubsection{Acupuncture}

Acupuncture has long been used in China and Taiwan, and its analgesic effects appear faster compared to conventional analgesic therapies, furthermore, they are without any complications. In a study by Lee et al. concerning the effects of acupuncture on pain management for renal colic, it was determined that the effect of acupuncture compared to avofortan was quicker; hence, acupuncture was introduced as an alternative method for renal colic pain control. Acupuncture plays its role by increasing endogenous opiate levels in the cerebrospinal fluid (9).

\section{Conclusions}

Nowadays, opiates and NSAIDs are used in most countries for the control of renal colic pain. Knowing that these drugs have side effects, the administration of alternative therapies would appear to be inevitable. In the present review we presented almost all possible treatments for renal colic. However, more studies are needed to be conducted on the use of alternative therapies in renal colic. Some medications such as lidocaine and papaverine have worked well in patients resistant to conventional therapies; however, as inadequate evidence is available in this 
regard, further studies are needed. Furthermore, the effectiveness of rapid pain control of renal colic and complications resulting from these medications need to be reviewed in future trials.

\section{Acknowledgements}

We would like to thank Dr. Kamaleddin Hassanzadeh, Associate Professor of Urology at the Department of Urology, Tabriz University of Medical Sciences, Tabriz, Iran and Dr. Hassan Vaezi, Emergency Medicine at the Department of Emergency Medicine, Tabriz University of Medical Sciences, Tabriz, Iran for their help in the preparation of this manuscript.

\section{Authors' contributions}

Study concept and design: Samad EJ Golzari, Hassan Soleimanpour and Saeid Safari; Drafting of the manuscript: Farzad Rahmani, Nahid Zamani Mehr, Yaghoub Heshmat and Hanieh Ebrahimi Bakhtavar.

\section{Financial Disclosure}

The authors declare they have no financial disclosure.

\section{Funding/Support}

This article was not supported by any funding organization. There was no sponsor for this work.

\section{References}

1. Portis AJ, Sundaram CP. Diagnosis and initial management of kidney stones. Am Fam Physician. 2001;63(7):1329-38.

2. Holdgate $\mathrm{A}, \mathrm{Oh} \mathrm{CM}$. Is there a role for antimuscarinics in renal colic? A randomized controlled trial.J Urol. 2005;174(2):572-5.

3. Soleimanpour H, Hassanzadeh K, Vaezi H, Golzari SE, Esfanjani RM, Soleimanpour M. Effectiveness of intravenous lidocaine versus intravenous morphine for patients with renal colic in the emergency department. BMC Urol. 2012;12:13.

4. Holdgate A, Pollock T. Nonsteroidal anti-inflammatory drugs (NSAIDs) versus opioids for acute renal colic. Cochrane Database Syst Rev. 2005;(2):CD004137.

5. Dellabella M, Milanese G, Muzzonigro G. Randomized trial of the efficacy of tamsulosin, nifedipine and phloroglucinol in medical expulsive therapy for distal ureteral calculi.JUrol. 2005;174(1):167-72.

6. Phillips E, Hinck B, Pedro R, Makhlouf A, Kriedberg C, Hendlin K, et al. Celecoxib in the management of acute renal colic: a randomized controlled clinical trial. Urology. 2009;74(5):994-9.

7. Safdar B, Degutis LC, Landry K, Vedere SR, Moscovitz HC, D’Onofrio G. Intravenous morphine plus ketorolac is superior to either drug alone for treatment of acute renal colic. Ann Emerg Med. 2006;48(2):173-81

8. Holdgate A, Pollock T. Systematic review of the relative efficacy of non-steroidal anti-inflammatory drugs and opioids in the treatment of acute renal colic. BMJ. 2004;328(7453):1401.

9. Davenport K, Timoney AG, Keeley FX. Conventional and alternative methods for providing analgesia in renal colic. BJU Int 2005;95(3):297-300.

10. Serinken M, Karcioglu O, Turkcuer I, Ozkan HI, Keysan MK, Bukiran A. Analysis of clinical and demographic characteristics of patients presenting with renal colic in the emergency department. BMC Res Notes. 2008;1:79.

11. Iguchi M, Katoh Y, Koike H, Hayashi T, Nakamura M. Randomized trial of trigger point injection for renal colic. Int J Urol. 2002;9(9):475-9.
12. Bektas F, Eken C, Karadeniz O, Goksu E, Cubuk M, Cete Y. Intravenous paracetamol or morphine for the treatment of renal colic: a randomized, placebo-controlled trial. Ann Emerg Med. 2009;54(4):56874.

13. Edwards JE, Meseguer F, Faura C, Moore RA, McQuay HJ. Single dose dipyrone for acute renal colic pain. Cochrane Database Syst Rev 2002;(4):CD003867.

14. Hazhir S, Ahmadi YA, Darabi JN. Intramuscular Tramadol versus Intramuscular Pethidine for Treatment of Acute Renal Colic. Urol J 2010;7:148-51.

15. Davenport K, Waine E. The Role of Non-Steroidal Anti-Inflammatory Drugs in Renal Colic. Pharmaceuticals. 2010;3(5):1304-1310.

16. Supervía A, Pedro-Botet J, Nogués X, Echarte JL, Mínguez S, Iglesias ML, et al. Piroxicam fast-dissolving dosage form vs diclofenac sodium in the treatment of acute renal colic: a double-blind controlled trial. BrJUrol.1998;81(1):27-30.

17. Labanaris AP, Kühn R, Schott GE, Zugor V. Perirenal hematomas induced by extracorporeal shock wave lithotripsy (ESWL). Therapeutic management. Sci World J.2007;7:1563-6.

18. Massó González EL, Patrignani P, Tacconelli S, García Rodríguez LA Variability among nonsteroidal antiinflammatory drugs in risk of upper gastrointestinal bleeding. Arthritis Rheum. 2010;62(6):1592 601.

19. Cohen E, Hafner R, Rotenberg Z, Fadilla M, Garty M. Comparison of ketorolac and diclofenac in the treatment of renal colic. Eur J Clin Pharmacol.1998;54(6):455-8.

20. Larkin GL, Peacock WF 4th, Pearl SM, Blair GA, D'Amico F. Efficacy of ketorolac tromethamine versus meperidine in the ED treatment of acute renal colic. Am J Emerg Med.1999;17(1):6-10.

21. Lafrance JP, Miller DR. Selective and non-selective non-steroidal antiinflammatory drugs and the risk of acute kidney injury. Pharmacoepidemiol Drug Saf. 2009;18(10):923-31.

22. Bleumink GS, Feenstra J, Sturkenboom MC, Stricker BH. Non-Steroidal Anti-Inflammatory Drugs and Heart Failure. Drugs. 2003;63:525534.

23. Kearney PM, Baigent C, Godwin J, Halls H, Emberson JR, Patrono C Do Selective Cyclo-Oxygenase-2 Inhibitors and Traditional Non-Steroidal Anti-Inflammatory Drugs Increase the Risk of Atherothrombosis? Meta-Analysis of Randomised Trials. BMJ. 2006;332:1302-1308

24. Serinken M, Eken C, Turkcuer I, Elicabuk H, Uyanik E, Schultz CH. Intravenous paracetamol versus morphine for renal colic in the emergency department: a randomised double-blind controlled trial.Emerg Med J. 2012;29(11):902-5.

25. Morgan S. Intravenous Paracetamol in Patients with Renal Colic Emerg Nurse J.2011;18(9):22-25.

26. Duggan ST, Scott LJ. Intravenous Paracetamol (Acetaminophen) Drugs.2009;69(1):101-113.

27. Göröcs TS, Lambert M, Rinne T, Krekler M, Modell S. Efficacy and tolerability of ready-to-use intravenous paracetamol solution as monotherapy or as an adjunct analgesic therapy for postoperative pain in patients undergoing elective ambulatory surgery: open, prospective study. Int J Clin Pract. 2009;63(1):112-20.

28. Grissa MH, Claessens YE, Bouida W, Boubaker H, Boudhib Kerkeni W, et al. Paracetamol vs piroxicam to relieve pain in renal colic. Results of a randomized controlled trial. Am J Emerg Med. 2011;29(2):203-6.

29. Lee SY, Lee WH, Lee EH, Han KC, Ko YK. The effects of paracetamol, ketorolac, and paracetamol plus morphine on pain control after thyroidectomy. Korean J Pain. 2010;23(2):124-30.

30. Douglas C, Dillon DC, Gibbs MA. Local and Regional Anesthesia. In Judith E. Tintinalli, editor. Tintinalli: Tintinalli's Emergency Medicine. China: McGraw-Hill; 2011. pp. 270-283.

31. Ferrini RA, Paice J. How to Initiate and Monitor Infusional Lidocaine for Severe and/or Neuropathic Pain. J. Supportive Oncol. 2004;2:9094.

32. Soleimanpour H, Hassanzadeh K, Mohammadi DA, Vaezi H, Esfanjani RM. Parenteral lidocaine for treatment of intractable rena colic: a case series. J Med Case Rep. 2011;29(5):256.

33. Samuels LA. Pharmacotherapy Update: Hyoscine Butylbromide in the Treatment of Abdominal Spasms. Clinical Medicine:Therapeutics. 2009;1:647-655.

34. Kheirollahi AR, Tehrani M, Bashashati M. A comparison of the Effect 
of Intranasal Desmopressin and Intramuscular Hyoscine N-Butyl Bromide Combination with Intramuscular Hyoscine N-Butyl Bromide Alone in Acute Renal Colic. J Res Med Sci. 2010;15(4):214-8.

35. Khurram Sarfraz, Khubaib Shehzad, Irfan Ali Sheikh. Comparison between pain relief achieved by Diclofenac alone and the combination of Diclofenac and Hyoscine in the short-term treatment of ureteric colic. PakArmed Forces Med J. 2008;58(2):136-40.

36. Song SW, Kim K, Rhee JE, Lee JH, Seo GJ, Park HM. Butylscopolammonium bromide does not provide additional analgesia when combined with morphine and ketorolac for acute renal colic. Emerg Med Australas. 2012;24(2):144-50.

37. Boubaker H, Boukef R, Claessens YE, Bouida W, Grissa MH, Beltaief K, et al. Phloroglucinol as an adjuvant analgesic to treat renal colic. Am JEmerg Med. 2010;28(6):720-3.

38. Sakhare RS, Roge AB, Jadhav SB, Ingole PG, Ahmed J, Jadhav AB. Two Wavelength Method for Estimation of Drotaverine Hydrochloride and Mefenamic Acid in their Combined Tablet Dosage Form. Int J Pharm Tech Res. 2012;4(3):1288-1293.

39. Palea S, Robineau P, Rischmann P, Lluel P. Relaxant Effect of Drotaverine (No-Spa) in Human Isolated Ureteral Rings. Eur Urol. 2006;sup 5:32.

40. Romics I, Molnár DL, Timberg G, Mrklic B, Jelakovic B, Köszegi G, et al. The effect of drotaverine hydrochloride in acute colicky pain caused by renal and ureteric stones. BJU Int. 2003;92(1):92-6.

41. Choe H, Lee YK, Lee YT, Choe H, Ko SH, Joo CU, et al. Papaverine blocks hKv1.5 channel current and human atrial ultrarapid delayed rectifier K+ currents. J Pharmacol Exp Ther. 2003;304(2):706-12.

42. Asgari SA, Asli MM, Madani AH, Maghsoudi PA, Ghanaei MM, Shakiba $\mathrm{M}$, et al. Treatment of loin pain suspected to be renal colic with papaverine hydrochloride: a prospective double-blind randomised study. BJU Int. 2012;110(3):449-52.

43. Snir N, Moskovitz B, Nativ O, Margel D, Sandovski U, Sulkes J, et al. Papaverine hydrochloride for the treatment of renal colic: an old drug revisited. A prospective, randomized study.JUrol. 2008;179(4):1411-4.

44. Yencilek F, Aktas C, Goktas C, Yilmaz C, Yilmaz U, Sarica K. Role of papaverine hydrochloride administration in patients with intractable renal colic: randomized prospective trial. Urology. 2008;72(5):987-90.
45. Barzegarnezhad A, Firouzian A, Emadi SA, Mousanejad N, Bakhshal R. The Effects of Local Administration of Aminophylline on Transureteral Lithotripsy. Adv Uro. 2012

46. Nickels TJ, Schwartz AD, Blevins DE, Drummond JT, Reed GW, Wilson DF. Effect of theophylline and aminophylline on transmitter release at the mammalian neuromuscular junction is not mediated by cAMP. Clin Exp Pharmacol Physiol. 2006;33(5-6):465-70.

47. Djaladat H, Tajik P, Fard SA, Alehashemi S. The Effect of Aminophylline on Renal Colic: A Randomized Double Blind Controlled Trial. South Med J. 2007;100(11):1081-4.

48. Kekec Z, Yilmaz U, Sozuer E. The Effectiveness of Tenoxicam Versus Isosorbide Dinitrate Plus Tenoxicam in the Treatment of Acute Renal Colic. BJU Int. 2000;85:783-5.

49. Hussain Z, Inman RD, Elves AW, Shipstone DP, Ghiblawi S, Coppinger SW. Use of glyceryl trinitrate patches in patients with ureteral stones: a randomized, double-blind, placebo-controlled study. Urology. 2001;58(4):521-5.

50. Porpiglia F, Destefanis P, Fiori C, Fontana D. Effectiveness of Nifedipine and Deflazacort in the Management of Distal Ureteric Stones. Urology. 2000;56:579-82.

51. Sümer A, Kaynar M, Topbas E, Hassan MA, Gürbüz R. Comparison of the Therapeutic Effects of Diclofenac Sodium, Prednisolone and an Alpha Blocker for the Treatment of Renal Colic. Turkish J Urology. 2012;38(1):23-28.

52. Nuss GR, Rackley JD, Assimos DG. Adjunctive Therapy to Promote Stone Passage. Rev Urol. 2005;7:67-74.

53. Yilmaz E, Batislam E, Basar MM, Tuglu D, Ferhat M, Basar H. The Comparison and Efficacy of 3 Different Alpha1-Adrenergic Blockers for Distal Ureteral Stones. J Urol. 2005;173:2010-2.

54. Parsons JK, Hergan LA, Sakamoto K, Lakin C. Efficacy of Alpha-Blockers for the Treatment of Ureteral Stones.JUrol. 2007;177(3):983-7.

55. Dellabella M, Milanese G, Muzzonigro G. Efficacy of Tamsulosin in the Medical Management of Juxtavesical Ureteral Stones. J Urol. 2003;170: 2202-5.

56. Kober A, Dobrovits M, Djavan B, Marberger M, Barker R, Bertalanffy P, et al. Local Active Warming: An Effective Treatment for Pain, Anxiety and Nausea Caused by Renal Colic. JUrol.2003;170:741-4. 